\title{
DISCLAIMER
}
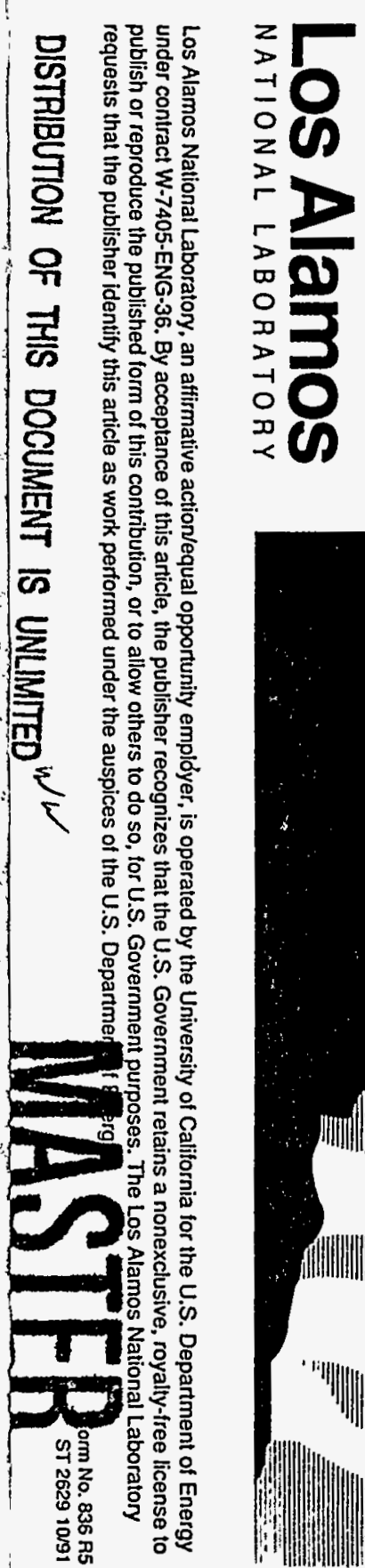

This report was prepared as an account of work sponsored by an agency of the United States Government. Neither the United States Government nor any agency thereof, nor any of their employees, makes any warranty, express or implied, or assumes any legal liability or responsibility for the accuracy, completeness, or usefulness of any information, apparatus, product, or process disclosed, or represents that its use would not infringe privately owned rights. Reference herein to any specific commercial product; process, or service by trade name, trademark, ence herein to any specific commercial product; process, or service by trade name, trademark,
manufacturer, or otherwise does not necessarily constitute or imply its endorsement, recommendation, or favoring by the United States Government or any agency thereof. The views and opinions of authors expressed herein do not necessarily state or reflect those of the United States Government or any agency thereof.
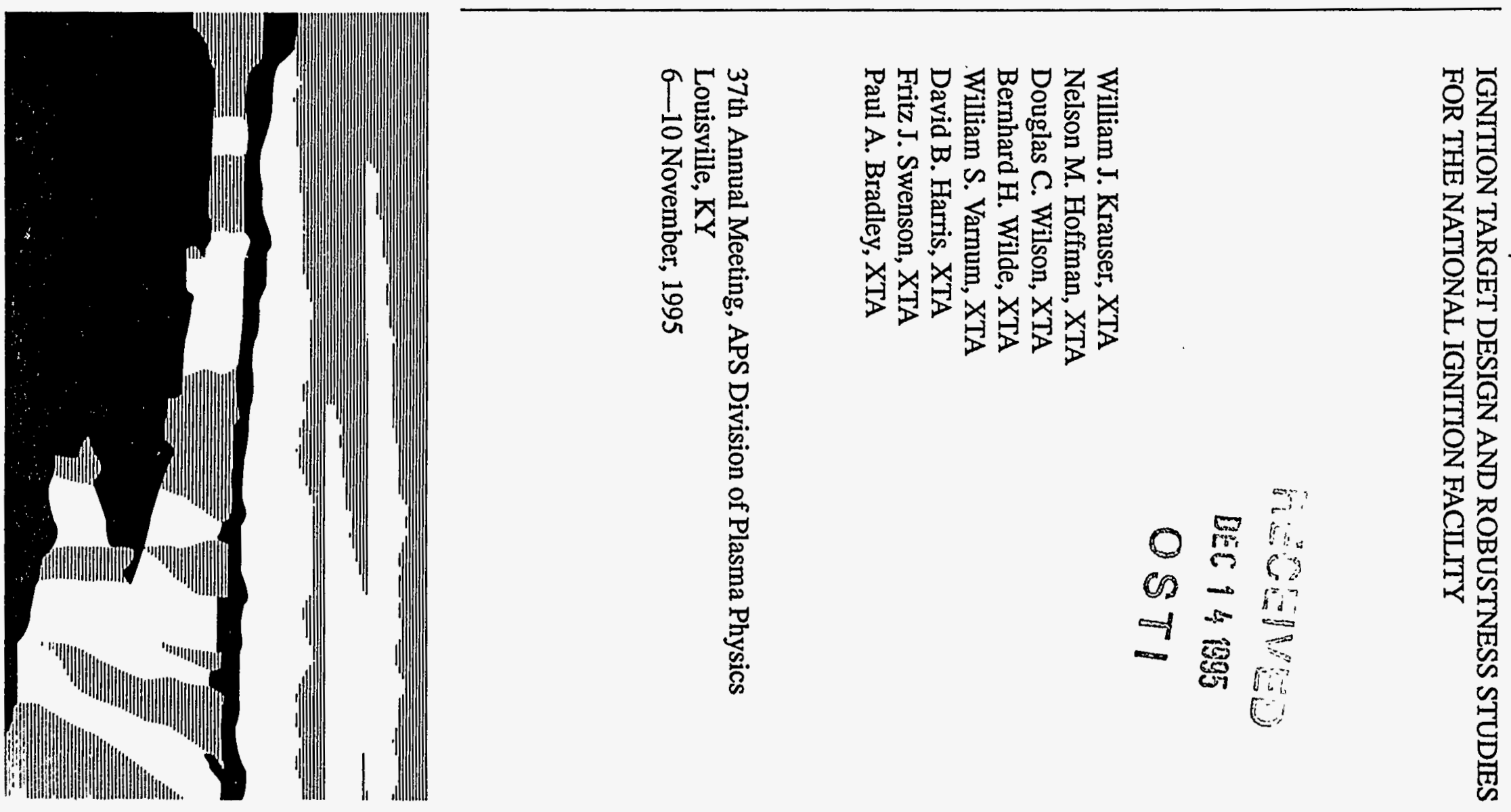


\section{DISCla IMIER}

Portions of this document may be illegible in electronic image products. Images are produced from the best available original document. 
Ignition target design and robustness studies for the National Ignition Facility

William J. Krauser, Nelson M. Hoffman, Douglas C. Wilson, Bernhard H. Wilde, William S. Varnum, David B. Harris, Fritz J. Swenson, Paul A. Bradley Los Alamos National Laboratory, Los Alamos, NM 87545

Steven W. Haan, Stephen M. Pollaine, Alan S. Wan, Juan C. Moreno, Peter. A. Amendt Lawrence Livermore National Laboratory, Livermore, CA 94550

Recent results are presented from two-dimensional LASNEX calculations of the indirectly driven hohlraum and ignition capsules proposed for the National Ignition Facility (NIF). The calculations concentrate on two capsule designs, the baseline design which has a bromine-doped plastic ablator, and the beryllium design which has a copperdoped beryllium ablator. Both capsules have a cryogenic fuel layer. Primary emphasis in these calculations is placed upon robustness studies detailing various sensitivities. These studies fall naturally into two categories, those performed with integrated modeling where the capsule, hohlraum, and laser rays all are modeled simultaneously with the laser power levels as the only energy input, and those performed in a capsule-only mode where an externally imposed drive is applied to the exterior of the ignition capsule and only the capsule performance is modeled. Integrated modeling calculations address sensitivities to, e.g., the laser pointing; among other things, capsule-only calculations address yield degradation due to the growth of hydrodynamic instabilities seeded by initial surface roughnesses on the capsules. Limitations of the calculational models and directions for future research are discussed. The results of the robustness studies performed to date enhance the authors' confidence that the NIF can achieve ignition and produce $10-15 \mathrm{MJ}$ of capsule yield with one or more capsule designs. 


\section{INTRODUCTION}

The inertial confinement fusion (ICF) program has the goal of producing useful thermonuclear burn from a deuterium-tritium (DT) target driven with laser or ion beams $[1,2]$. The National Ignition Facility (NIF) is a proposed laser system believed capable of producing ignition and intermediate gain. As currently proposed, the NIF laser is a 192 beam frequency tripled Nd:glass system providing a peak power of $500 \mathrm{TW}$ and a total energy of 1.8 MJ. The baseline NIF ignition target design is indirect drive. In this technique laser light is utilized to heat the interior walls of a high- $\mathrm{Z}$ cylindrical enclosure, or hohlraum. The hohlraum serves to isotropize and thermalize the initially highly localized and non-Planckian X-rays created when the laser light is absorbed. A spherical capsule in the hohlraum is irradiated nearly uniformly from almost all directions by the $\mathrm{x}$ rays filling the hohlraum. A highly symmetric ablation-driven implosion of the capsule results.

The baseline capsule is cryogenic with a DT ice shell surrounding a central DT gas region. The ice, in tum, is surrounded by an ablator. The hohlraum radiation temperature peaks at about $300 \mathrm{eV}$ and has a carefully shaped pre-pulse which keeps the fuel and the ablator on a low adiabat during the long, high-convergence-ratio implosion $(-20-35$, and defined as the initial outer radius of the ablator, divided by the ignition-time hotspot radius). For the baseline capsule and hohlraum, the peak hohlraum temperature cannot be pushed much higher than $300 \mathrm{eV}$ because the hohlraum would fill too rapidly with plasma blown off from the hohlraum wall, which prevents subsequent laser beam propagation within the hohlraum. Also, laser-plasma instabilities become more severe at the higher intensities needed to drive a hohlraum to higher temperature. At a lower peak temperature, a capsule implosion with a higher convergence ratio (i.e., a thinner ice shell at larger initial radius) would be required to achieve the same fuel density and yield. In order not to quench the ignition, there must be little mixing of the ignition hotspot with the cooler main fuel. The higher the convergence ratio, however, the larger the growth of inevitable 
initial perturbations on, for example, the ice-gas interface. If this growth is too large, catastrophic hotspot mixing will occur and the capsule will fail to ignite. The baseline hohlraum and capsule design occupies a prudent compromise location in this parameter space. It is expected to produce $10-15 \mathrm{MJ}$ of yield.

Design issues and modeling studies of the baseline capsule and other similar targets have been addressed in detail [3]. These calculations, bolstered by extensive experimental campaigns associated with unresolved issues or poorly modeled phenomena, have provided the basis for continuing national support for the NIF. Ignition on the NIF, however, is not a certainty. To better quantify the probability of ignition, we have concentrated our calculational effort during the past year on wide-ranging robustness studies, both of the baseline target and various alternative targets. Results of these calculations, carried out at Los Alamos National Laboratory (LANL) and at Lawrence Livermore National Laboratory (LLNL), are presented below.

\section{MODELING REQUIREMENTS AND TECHNIQUES}

In designing or assessing the performance of NIF targets, we need to model a wide variety of physical phenomena that occurs during the course of the implosion. Examples include the refraction, reflection, and absorption of laser light passing through the spatially and temporally varying laser entrance holes (LEH's); the filling of the hohlraum with plasma blown off from the hohlraum wall; laser-plasma interactions including instability seeding and growth; the spot motion of the laser absorption region; the time dependent emission of $\mathrm{x}$-rays from the laser-heated plasma, their absorption on and re-radiation from the hohlraum walls, and final absorption in the capsule ablator; the spatial and temporal variation of the capsule ablation; the capsule implosion and short-wavelength hydrodynamic instability growth and mixing within the capsule; and thermonuclear burn. No single code exists today which is capable of modeling simultaneously all of these phenomena, some of which require a three-dimensional (3-D) treatment. 
The best calculations we can do currently are performed in 2-D with the Lagrangian radiation-hydrodynamics and fusion burn code LASNEX [4]. These calculations fall naturaliy into two categories, those performed with integrated modeling where the capsule, hohlraum, and laser rays all are modeled simultaneously with the laser power levels as the only energy input, and those performed in a capsule-only mode where an externally imposed drive is applied to the exterior of the ignition capsule and only the capsule performance is modeled.

In the integrated calculations, each of which requires the equivalent of $50-500$ hours of time on a Cray-YMP, we generally use the best available numerical packages for laser ray tracing [5], radiation transport, non-local thermodynamic equilibrium (non-LTE) atomic physics [6] in the hohlraum wall, hydrodynamics (mostly Lagrangian, but with an Eulerian or semi-Eulerian rezoning treatment $[7,8]$ for material in or near the LEH's), and fusion burn. This comprehensive but computationally intensive technique was developed over the last several years and models many of the phenomena listed above [3].

Nevertheless the calculations are incomplete:

(i) The calculation of colliding low density plasmas is performed with Lagrangian hydrodynamics and does not model plasma interpenetration. Under conditions where this phenomenon may be important, we utilize separate particle-in-cell calculations to model the plasma behavior.

(ii) Laser plasma instabilities such as stimulated Brillouin and Raman scattering (SBS and SRS) are not modeled explicitly. However, the integrated calculations of the baseline target use only approximately $1.4 \mathrm{MJ}$ of laser energy, leaving $0.4 \mathrm{MJ}$ of the NIF laser design energy to account for these and other losses.

(iii) Capsule mixing and yield degradation due to short scale length hydrodynamic instability growth during the implosion must be modeled separately in capsule-only calculations [9].

The techniques and physics packages used in integrated modeling of the NIF have 
been used in modeling relevant NOVA experimental data $[10,11]$. The generally excellent agreement between those calculations and the data increases our confidence that integrated modeling techniques and code physics packages are adequate.

Capsule-only calculations typically are as lengthy as the integrated calculations. In these studies the capsule is resolved in both angle and radius with zoning substantially finer than that used in most integrated calculations. Surface perturbations with realistic spectra of wavelengths and amplitudes derived from experimental measurements are applied to the inside ice surface, the outside ablator surface, or both. These are the most unstable interfaces. These calculations are driven by an externally imposed radiative flux on or near the capsule ablator surface. The drive can be uniform in angular position about the capsule, or it can have a time-varying angular dependence. Frequently the angular dependence is derived from previous integrated modeling simulations which can resolve long-wavelength asymmetries arising from the radiation drive asymmetry in the hohlraum. As the implosion proceeds, the initially small perturbations grow in a complicated, non-linear fashion since the flow is unstable.

In both the integrated modeling and capsule-only calculations, the simulation typically runs through the entire capsule implosion, the capsule ignition, and the subsequent thermonuclear burn and disassembly of the DT fuel. There is usually enough distortion of the Lagrangian mesh within the fuel, particularly in the capsule-only calculations, that some rezoning $[7,8]$ is required during the simulations to allow these calculations to continue through the time of fusion burn.

\section{DESCRIPTION OF HOHLRAUM AND IGNITION TARGETS}

There are many possible hohlraum and ignition capsule designs. We have focussed most of our work on one basic hohlraum design and on two different capsule designs (see Fig. 1). The baseline hohlraum is a $9.49 \mathrm{~mm}$ long by $2.76 \mathrm{~mm}$ radius gold cylinder, $40 \mu \mathrm{m}$ thick, with $1.39 \mathrm{~mm}$ radius LEH's on either end. LANL calculations include a $10 \mu \mathrm{m}$ thick 
plastic liner around the lip of the LEH to help keep it open. The hohlraum is filled with a $50-50$ (atomic) mixture of hydrogen-helium gas at a density of $0.833 \mathrm{mg} / \mathrm{cm}^{3}$. The mixture of gases reduces SBS [12]. The gas is contained within the hohiraum by $1 \mu \mathrm{m}$ thick polyamide foils covering each LEH.

The capsule uses either a $160 \mu \mathrm{m}$ thick polystyrene (plastic) ablator doped with bromine (this is the baseline capsule, also called the PT for "Point-design Target"), or a $155 \mu \mathrm{m}$ thick beryllium ablator doped with copper (the $300 \mathrm{eV}$ Be design). The ablator surrounds an $80 \mu \mathrm{m}$ thick DT ice layer with an inner radius of $0.87 \mathrm{~mm}$. The ablator dopant serves to reduce $\mathrm{x}$-ray preheat in the ablator, and in the PT it eliminates an unstable density step at the ablator-DT interface.

The hohlraum is driven by laser beams entering both LEH's. As shown in Fig. 1, the laser light coming in each LEH is in two cones, an inner cone which illuminates the hohlraum wall near the waist or equator of the capsule, and an outer cone which illuminates an area of the wall closer to the LEH. Each inner and outer cone is comprised of two sub-cones. This arrangement was chosen so that the time-dependent $\mathrm{P}_{2}$ component of the radiation incident on the capsule can be minimized by dynamically varying the relative power in the cones, a procedure called "beam phasing." IIt is customary to quantify the radiation drive asymmetry by expanding the radiative flux incident on the capsule in terms of spherical harmonics, which reduce to Legendre polynomials on the surface of a sphere having cylindrical symmetry. The independent variable in the expansion is the angle $\theta$, which is $0^{\circ}$ at the capsule pole and $90^{\circ}$ at the capsule equator. Left-right symmetry in the hohlraum ensures that the odd moments $P_{1}, P_{3}$, etc., in the expansion vanish. The $\mathrm{P}_{0}$ moment is the average flux incident on the capsule and is roughly proportional to the fourth power of the hohlraum radiation temperature. $\mathrm{P}_{\mathbf{2}}$ is a measure of the pole-to-equator asymmetry. Ideally, all but the $\mathrm{P}_{0}$ moment are zero.]

As discussed in Ref. 3, efficient capsule burn is achieved through a series of integrated calculations where the laser power levels, the beam pointing, and the beam phasing are 
adjusted until'a low entropy, highly symmetric implosion of the DT fuel is attained. This optimization of the capsule implosion and burn in integrated calculations is called "tuning." Tuned calculations of the plastic capsule design produce yields of $12 \mathrm{MJ}$ or more while the $300 \mathrm{eV}$ beryllium capsule produces about $15 \mathrm{MJ}$. For reference, Fig. 2 shows the ideal radiation temperature drive for the PT and a laser power profile from an integrated calculation that approximately generates this drive. The ideal drive consists of a foot, a second rise, a third rise, and, finally, a peak. The four shocks, each carefully timed and sized, that are generated in the capsule bring the fuel to a high average density but low entropy, except in the central ignition "hotspot."

\section{INTEGRATED MODELING ROBUSTNESS STUDIES}

We examined the sensitivity of the PT to variations in laser parameters such as the power during the foot, the peak power, the timing of the rises, and the pointing of the inner and outer beams. For some of these variations, re-tuning was required. As shown in Table I, all of the sensitivities examined are consistent with the laser specifications. An entirely unexpected but pleasing observation was made during the course of these studies. For certain optimal pointings of the outer cone, no beam phasing is required to achieve symmetric, high yield implosions. In other words, the fraction of the total laser power going to the inner cone can be constant at about $32.5 \%$ for the duration of the laser pulse. If this fraction is reduced to $28 \%$ or increased to $34 \%$ the capsule yield is halved. Moving the outer beams a modest $100 \mu \mathrm{m}$ towards the LEH necessitates a return to beam phasing in order to restore implosion symmetry and yield.

Another method of achieving good capsule yield with no beam phasing is to separate the two sub-cones which together comprise the outer cone. This 3-cone illumination scheme results in more uniform heating (compared to the baseline 2-cone scheme) of the hohlraum wall near the LEH which is reflected in a reduction of the average $P_{4} / P_{0}$ moment of the radiation flux incident on the capsule. 
We examined the sensitivity of the PT to changes in the length of the hohlraum and the density of the H-He gas fill. As shown in Fig. 3, increasing the hohlraum length by $500 \mu \mathrm{m}$ and increasing the gas density to $1.2 \mathrm{mg} / \mathrm{cm}^{3}$ provides a much wider path at the time of peak laser power for the inner cone laser rays to reach the hohlraum wall near the capsule equator. This wider path reduces undesirable laser absorption and refraction in the gold blow-off under the outer laser cone and in the ablated material from the capsule. The calculation required $1.42 \mathrm{MJ}$ of laser energy in order to keep the peak hohlraum temperature at $300 \mathrm{eV}(1.34 \mathrm{MJ}$ is required in the baseline hohlraum design). At much higher gas densities $\left(-3 \mathrm{mg} / \mathrm{cm}^{3}\right)$ undesirable hydro-coupling occurs between the gas and the PT ablator near the pole. The resulting implosion is unacceptably asymmetric.

We addressed the adequacy of several important physics approximations and numerical techniques used in integrated calculations:

(i) Our non-LTE atomic physics package models the time dependence of the level populations in the laser-irradiated gold plasma atoms. Use of this non-LTE package shows less radiation emission from the laser deposition regions, more gold blow-off into the hohlraum, and lower hohlraum temperatures, given constant laser power, compared to the use of the less sophisticated, but faster running LTE atomic physics treatment. Since the non-LTE calculations are more pessimistic, they are used almost exclusively in other robustness studies.

(ii) Large changes in the electron conduction flux limiter used in the hohlraum gas only modestly affect the hohlraum or the capsule yield, although re-tuning is required.

(iii) We have benchmarked our best available radiation transport package and have shown that is adequate.

(iv) We have demonstrated, using $2^{\circ}$ rather than the standard $5^{\circ}$ angular zoning in the capsule, that our standard zoning results in reasonably converged simulations. Similar fuel configurations at ignition time and similar yields were observed in the comparison.

Finally, using three plastic ablator targets that are geometric scales of the PT, we 
demonstrated adequate symmetry and good ignition and burn in integrated calculations having input laser energies ranging from the full $1.8 \mathrm{MJ}$ design energy down to less than 1 $\mathrm{MJ}$ (see Fig. 4). In addition to the burn achieved in the $300 \mathrm{eV}$ Be design, we previously achieved good burn in a different $\mathrm{Be}$ design driven at $250 \mathrm{eV}$ [3].

\section{CAPSULE-ONLY ROBUSTNESS STUDIES}

Recent evidence from theory and experiment $[13,14]$ points to a picture of late-stage instability in ICF implosions in which unstable interfaces are deformed into a network of polygonal bubbles surrounded by spike sheaths during the acceleration phase of the implosion. The bubbles occur with a range of sizes, but there is a dominant scale characterizing the largest and therefore most rapidly growing bubbles. The implication is that instability can disrupt these flows, not necessarily by fine-scale mixing, but by largescale effects that can prevent ignition by interfering with the compressional heating of the fusion fuel, by increasing the surface area through which heat is lost from the fuel, or by reducing the effectiveness of $\alpha$-particle trapping.

To model the bubble-network picture of hydrodynamic instability, we have performed direct numerical simulations (DNS) in which the unstable flow is represented with as much detail and as few modeling approximations as possible, given the constraints of finite machine memory and two-dimensionality. In the calculation, many spherical harmonic perturbation modes (up to 24 thus far) are imposed on each interface of interest, with realistic amplitude spectra, and a 90-degree quadrant of the capsule is represented to allow proper boundary conditions for all modes simultaneously. We do not need to model capsule burn performance separately from capsule stability, since the yield and other attributes of the capsule are calculated at the same time as the perturbation growth. Thus with DNS we avoid assumptions made in other approaches $[15,16,17]$ about mode saturation or enhanced heat fiow, since we calculate these effects explicitly and have no need for model parameters that adjust their magnitudes. 
Two-dimensionality introduces at least two shortcomings in the calculations, one of which we can compensate for, while the other we cannot. First, a 2-D surface, because of its axial symmetry, is inherently less rough than a 3-D surface with the same spherical harmonic spectrum. To try to compensate for this effect in our calculations, we modify the relative amplitude of all modes in the 2-D spectrum by the factor $\sqrt{2 l+1}$, while keeping the total root-mean square (RMS) surface roughness $\sigma$ constant. This modified spectrum we call a "bandwidth-equivalent-RMS" (BER) spectrum, because it has not only the same total $\sigma$ as the 3-D spectrum which it is supposed to represent, but each mode, or band of modes, makes the same relative contribution to the total $\sigma$ as in the 3-D spectrum.

The other shortcoming arises because of the fact that 2-D bubbles grow more slowly than 3-D bubbles. This is called the "shape effect" [14], and was pointed out by Layzer [18]. The terminal velocity of a bubble is about 50\% higher in 3-D than in 2-D, for large density ratios. There is no obvious way to compensate for the shape effect in a 2-D calculation, so a resolution must await 3-D calculations. In the meantime, we take the viewpoint that 2-D calculations furnish a lower limit to the magnitude of the disruptive effects of a perturbation with a given initial $\sigma_{0}$, and therefore our conclusions about the maximum tolerable $\sigma=\sigma_{\text {crit }}$ are actually upper limits to its value.

Another issue raised by our DNS is that of Lagrangian mesh distortion. Because the mode amplitudes are finite, the perturbation at an interface can eventually grow to seriously disturb the capsule implosion. In this case the distinction between zeroth-order and first-order flow is lost, distinct bubbles and spikes form in the density structure, the mesh becomes greatly deformed; the code's time step drops to extremely small values, and the calculation halts.

To continue the calculation requires rezoning, that is, defining a new, more regular mesh and mapping the physical variables from the old deformed mesh to the new regular mesh. In defining the new mesh, LANL calculations employ a remeshing technique due to Brackbill and Saltzman [7] which allows the mesh to adapt to steep gradients in physical 
variables, solving an elliptical equation to define the new mesh lines using the old mesh as. a boundary condition. Then a 2nd-order-accurate technique due to Scannapieco [8] is used for the remapping of variables. Repeated application of the rezoning procedure allows the calculation to follow flow patterns which are quite turbulent. It is clear, however, that the calculated performance of a capsule, in particular its yield, can be affected by the exact prescription for rezoning (e.g., the time at which rezoning begins, the frequency with which it is carried out, and the degree to which the new mesh is allowed to approach the exact Brackbill-Saltzman solution). The sensitivity is greatest near "cliffs" in initial surface roughness, i.e., when $\sigma_{0}$ is near $\sigma_{\text {crit }}$. The effect is to make the location of the cliff uncertain to some degree. We are currently assessing the relative accuracy of the various prescriptions for rezoning, through comparison to experiments and theory. In the meantime, we will simply conclude that rezoning is required to allow these calculations to continue through the time of fusion burn of the capsule, but introduces some uncertainty into the results.

We have applied DNS to several ignition capsule designs in order to specify the maximum tolerable $\sigma_{0}$ of various surfaces in the capsule. Figure 5 shows a typical initial perturbation of the inner DT ice interface, where the perturbation is the sum of 24 spherical harmonic modes $(l=2,4,6, \ldots, 48)$. The modal amplitudes are given by a BER spectrum obtained by scaling a 1-D Fourier analysis of cryogenic DT data to a 2-D power spectrum, and then applying the BER enhancement discussed earlier.

Figure 6 shows a comparison of DNS yields for the PT and $300 \mathrm{eV}$ Be capsules from an early series of relatively crude calculations where a non-BER spectrum consisting of only 12 modes $(l=4,8,12, \ldots, 48)$ was imposed on the outer surface of the ablators only. Both capsules are relatively insensitive to $\sigma$, the initial ablator RMS amplitude, until $\sigma$ exceeds about $40 \mathrm{~nm}$. This value is comfortably above the current state-of-the-art fabrication capability for plastic and Be ablators, about $20 \mathrm{~nm}$ and $4 \mathrm{~nm}$, respectively.

Figure 7 shows a similar comparison of performance, again from relatively crude 
calculations, but here the non-BER perturbation spectrum, consisting of 8 modes $(l=12,16,20, \ldots, 40)$ was imposed on the inner ice surface only. The $300 \mathrm{eV}$ Be capsule shows considerably greater tolerance for ice surface roughness than the PT. Until very recently, the state-of-the-art fabrication capability for the ice surface RMS roughness was $1.44 \mu \mathrm{m}$. The latest measured RMS roughness is about $1 \mu \mathrm{m}$. It appears that both capsules have some performance margin above the current fabrication capability, at least in calculations where only the ice surface is perturbed, but the margin for the Be capsule is much more comfortable.

For the PT design we have performed calculations in which both the ablator and the inner ice surface are perturbed with 24-mode BER spectra. We varied the amplitude of the initial DT ice perturbation in an attempt to identify the maximum ice roughness that the capsule can tolerate in the absence of radiation asymmetry. These were done with a fixed ablator surface roughness of $20 \mathrm{~nm}$ and with $0.5^{\circ}$ angular zoning. Modes $l=2,4,6, \ldots, 48$ were imposed on the DT ice and modes $l=4,8,12, \ldots, 96$ were imposed on the ablator. Figure 8 shows the resulting yield as a function of $\sigma_{\mathrm{DT}}$ the initial DT ice RMS amplitude, as filled circles. The yield of the PT capsule is relatively insensitive to $\sigma$ until it reaches a critical value, $\sigma_{\mathrm{DT}, \text {,rit }}$ above which the yield falls dramatically, indicating a failure to ignite. These calculations place $\sigma_{\mathrm{DT} \text {,crit }}$ at about $3 \mu \mathrm{m}$. This is roughly comparable with what was found in the earlier DNS, discussed above, that used the non-BER perturbation with relatively high modes only $(l=12,16,20, \ldots, 40)$ and had no ablator roughness (circles in Fig. 7 or open triangles in Fig. 8). The shaded triangles in Fig. 8 show results for a 24 mode non-BER spectrum with low modes included $(l=2,4,6, \ldots, 48)$. The location of the cliff, $\sigma=\sigma_{\text {crit }}$ increases for the spectrum with low modes included, because low modes grow less rapidly than high modes and produce less disruption of the flow. The BER spectra have somewhat more contribution from high modes than the 24-mode non-BER spectra, yet a higher value of $\sigma_{\mathrm{DT}, \text { crit }}$. We attribute this to differences in the rezoning prescription between the BER and non-BER calculations. The scatter in calculated yields 
near the cliff for a given type of spectrum results from variations.in the prescription for rezoning.

To see if the $20 \mathrm{~nm}$ ablator perturbation plays a significant role in establishing the value of $\sigma_{\mathrm{DT} \text {, crit }}$, we did a calculation on the edge of the cliff without ablator surface perturbations. The resulting yield is plotted in Fig. 8 as lightly shaded circles. Interestingly, the yield did not increase or remain unchanged as one would expect but instead it decreased by $1 \mathrm{MJ}$ or about $40 \%$. We have two possible explanations for this. One is that this is an example of mode coupling between the perturbation placed on the ablator and that placed on the ice. The resulting yield can then increase or decrease depending on complicated interactions among the modes and their phases. Alternatively, this result could be compatible with the hypothesis that surface perturbations of this order play no significant role when $\sigma_{\mathrm{DT}}$ is large $(4 \mu \mathrm{m})$ and that the change in yield should be ascribed to chance, given the fact that capsules that marginally ignite are known to be sensitive to small changes in initial conditions. We have yet to establish which of these possibilities is most likely or dominant.

Another interesting outcome from these and other calculations is the demonstration that mode coupling between the ice and ablator does occur, and that the relative phases of the modes can be a significant factor in the outcome of a multimode dual perturbation calculation. We conclude that interpreting the results of such multimode calculations should be done with some caution as a fortuitous choice of phases could mask real problems.

Of course, the hard part of our work is relating our two-dimensional findings to real three-dimensional capsules. In our two-dimensional simulations we have imposed linear waves of varying amplitude and frequency onto the surfaces of our two-dimensional capsules and it is fairly easy to see that phasing (there are only two choices of phase, positive and negative, for our $90^{\circ}$ problems) plays a major role in determining how deep the largest spike is. In three-dimensional reality there will be a largest spike or deepest 
dimple somewhere on the surface and we need to know how deep that defect generally can be. Note that the sizes of the largest dimples are not necessarily correlated with the RMS perturbation size over the surface as a whole. Therefore, we need measurements of real capsules and we need to do calculations that will determine what size dimple is acceptable given the existence of one such dimple or many such dimples. Such calculations are currently being contemplated. They should be carried out in a $180^{\circ}$ sector to avoid the exactly opposed "mirror" dimple that is effectively present in a $90^{\circ}$ calculation.

Using the technique of direct nonlinear multimode instability simulations, we can now begin to examine the coupling of various asymmetries and departures from ideal driving conditions, simultaneously or taken a few at a time. For example, we can study the sensitivity of a capsule to variations in the hohlraum drive history, as is often done in 1-D, but now include the non-ideal circumstances of surface roughness and drive asymmetry. Figure 9 shows results of such a study in which the yield of the $300 \mathrm{eV}$ Be capsule is determined as a function of the temperature in the final drive pulse. Open circles show the standard 1-D result, which assumes perfect smoothness of all capsule surfaces. The filled circles show the effect of $20 \mathrm{~nm}$ RMS roughness on the outer surface of the ablator, somewhat above the limit of our current Be fabrication capability. Most of the capsule's ability to tolerate low drive is negated by surface roughness. Future studies examining sensitivity to drive variations will include drive asymmetry and perturbations on all surfaces simultaneously.

We used DNS to incorporate the low-mode radiation drive asymmetry, determined by an integrated hohlraum calculation, into our instability calculations to examine its coupling to surface perturbations. We used BER surface perturbation spectra for surface perturbations on the outer plastic capsule surface and on the inner DT ice surface. The RMS roughness for the outer surface was taken to be $20 \mathrm{~nm}$ and the RMS roughness for the ice surface was taken to be $1.44 \mu \mathrm{m}$, values matching then current fabrication capabilities for the two surfaces (ice RMS roughnesses are now about $1 \mu \mathrm{m}$ ). A clean 1-D 
calculation of the yield of the PT capsule using the radial rezoning procedure necessary for the two-dimensional calculations gives 15.2 MJ. The equivalent yield for the full twodimensional calculation with both radiation asymmetry and surface perturbations is 9.6 MJ. Much of the short-wavelength structure one sees in the fuel at ignition time for surface perturbations alone disappears when the realistic drive asymmetries are included.

The most recent and comprehensive DNS of the PT incorporated low mode radiation drive asymmetry determined by an integrated hohlraum calculation, but with added variations representing uncertainties or errors in the laser operation and hohlraum behavior. In particular, up to 5\% RMS in $\mathrm{P}_{2}(\mathrm{t}), 1.5 \%$ RMS in $\mathrm{P}_{4}(\mathrm{t})$, and $5 \% \mathrm{P}_{0}(\mathrm{t})$ flux variations were superimposed on the nominal drive. Both the inner and outer ablator surfaces had BER perturbations. The ablator RMS roughness was $20 \mathrm{~nm}$ and the ice RMS roughness was $0.5 \mu \mathrm{m}$, somewhat better than our current $1 \mu \mathrm{m}$ fabrication capability. The simulation gave a satisfying $8 \mathrm{MJ}$ of capsule yield. A similar calculation with $1 \mu \mathrm{m}$ ice roughness is in progress.

The above calculations include about as much realistic two-dimensional detail as we are presently capable of modeling. They lack only in that they are not three dimensional. We are currently investigating and developing other codes to realize a capability for 3-D calculations.

\section{CONCLUSION}

Many additional calculations remain to be performed.

\section{ACKNOWLEDGMENT}

This work was performed under the auspices of the U. S. Department of Energy by the Los Alamos National Laboratory under contract W-7405-ENG-36 and by the Lawrence Livermore National Laboratory under contract W-7405-ENG-48. 


\section{REFERENCES}

1. J. H. Nuckolls, L. Wood, A. Thiessen, and G. B. Zimmerman, Nature 239, 129 (1972).

2. J. D. Lindl, R. L. McCrory, E. M. Campbell, Phys. Today 45(9), 32 (1992).

3. S. W. Haan, S. M. Pollaine, J. D. Lindl, L. J. Suter, L. V. Powers, R. L. Berger, E. Alley, G. Zimmerman, P. A. Amendt, W. K. Levedahl, R. A. Sacks, A. I. Shestakov, G. L. Strobel, S. Weber, W. Krauser, N. M. Hoffman, D. Wilson, and D. Harris, Phys. Plasmas 2, 2480 (1995).

4. G. B. Zimmerman and W. L. Kruer, Comm. Plasmas Phys. Controlled Thermonucl. Fusion 2, 51 (1975).

5. A. Friedman, Lawrence Livermore Laboratory Laser Program Annual Report No. UCRL-50021-83, 1983, pp. 3-51.

6. See National Technical Information Service Document No. UCRL-52276 [W. A. Lokke and W. H. Grasberger, in Lawrence Livermore National Laboratory Report No. UCRL-52276 (1977)]. Copies may be ordered from the National Technical Information Service, Springfield, Virginia 22161. G. B. Zimmerman and R. M. More, J. Quant. Spectrosc. Radiat. Transfer 23, 517 (1980); R. M. More, ibid. 27, 345 (1982).

7. J. Brackbill and J. Saltzman, Journal of Computational Physics 46, 342 (1982).

8. A. Scannapieco, Los Alamos National Laboratory Report No. LA-UR-82-2897 (1982).

9. N. M. Hoffman, D. C. Wilson, and W. S. Varnum, "Nonlinear multimode instability modeling for National Ignition Facility capsules," submitted to Proceedings of the 12th International Conference on Laser Interaction and Related Plasma Phenomena, Osaka, April $24-28,1995$.

10.A. A. Hauer, L. J. Suter, N. Delameter, D. Ress, L. Powers, G. Magelssen, D. Harris, O. Landen, E. Lindmann, W. Hsing, D. Wilson, P. Amendt, R. Theissen, R. Koop, D. . Phillion, B. Hammel, D. Baker, J. Wallace, R. Tumer, M. Cray, R. Watt, J. Kilkenny, and J. Mack, Phys. Plasmas 2, 2488 (1995).

11.E. L. Lindman and N. Delameter, "Modeling of drive-symmetry experiments in gas- 
filled hohlraums at NOVA," submitted to Proceedings of the 12th International Conference on Laser Interaction and Related Plasma Phenomena, Osaka, April 24 $28,1995$.

12.L. V. Powers, R. L. Berger, R. L. Kauffman, B. J. MacGowan, P. A. Amendt, C. A. Back, T. P. Bernat, S. N. Dixit, D. I. Eimerl, K. G. Estabrook, J. A. Harte, D. H. Kalantar, D. E. Klem, B. F. Lasinski, D. S. Montgomery, J. D. Moody, D. H. Munro, T. D. Shepard, L. J. Suter, R. E. Turner, and E. A. Williams, Phys. Plasmas 2, 2473 (1995).

13.B. A. Remington, S. V. Weber, S. W. Haan, J. D. Kilkenny, S. G. Glendinning, R. J. Wallace, W. H. Goldstein, B. G. Wilson, and J. K Nash, Phys. Fluids B 5, 2589 (1993). 14.J. P. Dahlburg, Bull. APS 39, 1576 (1994); D. Shvarts, Bull. APS 39, 1576 (1994). 15.D. H. Munro, Phys. Fluids B 1, 134 (1989).

16.S. W. Haan, Phys. Rev. A 39, 5812 (1989).

17.T. R. Dittrich, B. A. Hammel, C. J. Keane, R. McEachern, R. E. Turner, S. W. Haan, and L. J. Suter, Phys. Rev. Lett. 73, 2324 (1994).

18.D. Layzer, Astrophys. J. 122, 1 (1955). 


\section{TABLE I}

Table I. Sensitivity of the plastic ablator capsule to changes in the laser power, timing, cone-to-cone power balance, and pointing. Tolerable variations are defined as full-widthat-half-maximum in capsule yield, based on integrated calculations. Expected variations are preliminary estimates of system uncertainty, based on NIF laser specifications and experimental uncertainties.

\begin{tabular}{lll}
\hline \hline Laser parameter & Tolerable & Expected \\
\hline Power during foot & $30 \%$ & $<5 \%$ \\
Peak power & $35 \%$ & $<5 \%$ \\
Second rise timing & $500 \mathrm{ps}$ & $<100 \mathrm{ps}$ \\
Third rise timing & $500 \mathrm{ps}$ & $<100 \mathrm{ps}$ \\
$\begin{array}{l}\text { Duration of peak power } \\
\text { Inner beam power during foot } \\
\quad \text { total power fixed) }\end{array}$ & $800 \mathrm{ps}$ & $<100 \mathrm{ps}$ \\
$\begin{array}{l}\text { Inner beam power during peak } \\
\quad \text { (total power fixed) }\end{array}$ & $25 \%$ & $<5 \%$ \\
$\begin{array}{l}\text { Pointing of inner beams } \\
\text { Pointing of outer beams }\end{array}$ & $35 \%$ & $<5 \%$ \\
\hline \hline
\end{tabular}


Fig. 1. NIF capsules and hohlraum. The gold-walled, gas-filled hohlraum is driven by 192 laser beams (1.8 MJ, $500 \mathrm{TW})$ arrayed in inner and outer cones coming in from each side. The spherical capsule has a cryogenic DT fuel layer surrounded by a doped ablator. The capsule on the left is the plastic design (also called the PT) which has a $\mathrm{CH}$ ablator doped with $0.25 \% \mathrm{Br}$. The capsule on the right is the $300 \mathrm{eV}$ Be design with a Be ablator doped with $0.9 \% \mathrm{Cu}$.

Fig. 2. Laser power vs. time to drive the PT target (solid curve, left scale), and temperature vs. time optimal for the PT capsule (dashed curve, right scale). The shaped pulse prior to peak drive is needed to compress the target, increasing the pressure in a controlled way before applying peak power.

Fig. 3. Inner cone laser ray paths overlaid on the calculational mesh at peak laser power (14.5 ns) for the baseline hohlraum with length $0.95 \mathrm{~cm}$ and gas fill density $0.83 \mathrm{mg} / \mathrm{cm}^{3}$ (upper) and for a modified hohlraum with length $1.00 \mathrm{~cm}$ and gas fill density $1.20 \mathrm{mg} / \mathrm{cm}^{3}$ (lower). The modified hohlraum provides a wider path for the inner cone laser rays to reach the hohlraum waist.

Fig. 4. Yield and burn-weighted ion temperature for geometric scales of the PT. The lines show 1-D calculations of the capsule alone, in which linear dimensions and times are scaled together. For these, the horizontal scale is effective energy, that is, $1.35 \mathrm{MJ}$.times the scale factor cubed. The large dots are integrated calculations, plotted against the laser energy put into the simulation. For these, linear dimensions and times were scaled, and laser powers were scaled as the square of the scale factor. Hohlraum length and phasing were adjusted to recover symmetry. 
Fig. 5. A typical initial perturbation placed on the inner DT ice interface. The perturbation is the sum of 24 spherical harmonic modes $(l=2,4,6, \ldots, 48)$. The modal amplitudes are obtained by scaling a 1-D Fourier analysis of cryogenic DT data to a 2-D power spectrum, and then applying the BER enhancement. The RMS roughness $\sigma$ of this perturbation is $1.75 \mu \mathrm{m}$.

Fig. 6. Plastic and Be capsule yields as a function of ablator surface roughness, from 2-D multimode calculations with a non-BER, 12 mode $(l=4,8,12, \ldots, 48)$ initial spectrum. Both capsules tolerate ablator roughness up to about $40 \mathrm{~nm}$, comfortably above the $20 \mathrm{~nm}$ stateof-the-art fabrication capability for plastic and $4 \mathrm{~nm}$ capability for Be.

Fig. 7. Plastic and Be capsule yields as a function of DT ice surface roughness, from 2-D multimode calculations with a non-BER, 8 mode $(l=12,16,20, \ldots, 40)$ initial spectrum. The Be capsule tolerates substantially greater DT ice surface roughness than the plastic capsule. State-of-the-art fabrication capability for the ice surface is $1 \mu \mathrm{m}$ ("native"), or about $0.5 \mu \mathrm{m}$ ("heated") if the surface is fabricated and then heated briefly with RF. energy, a promising, but still experimental technique.

Fig. 8. Plastic capsule yield variations vs. DT ice inner surface roughness, for various perturbation spectra. Circles indicate calculations with BER spectra, and triangles show calculations with non-BER spectra. The filled circles indicate calculations with perturbations on both the ice and the ablator. The scatter in calculated yields near the critical surface roughness for a given type of spectrum results from variations in the prescription for rezoning.

Fig. 9. Drive sensitivity profiles for the Be capsule, plotted as calculated yield vs. multiplier applied to the final drive temperature. Open circles show standard 1-D results. 
The filled circles show the result of 2-D multimode calculations where the ablator is perturbed with an initial RMS roughness of $20 \mathrm{~nm}$, somewhat above the limit of our current Be fabrication capability. Most of the capsule's ability to tolerate low drive is negated by surface roughness. If the capsule is overdriven, ablator roughness has little effect. 

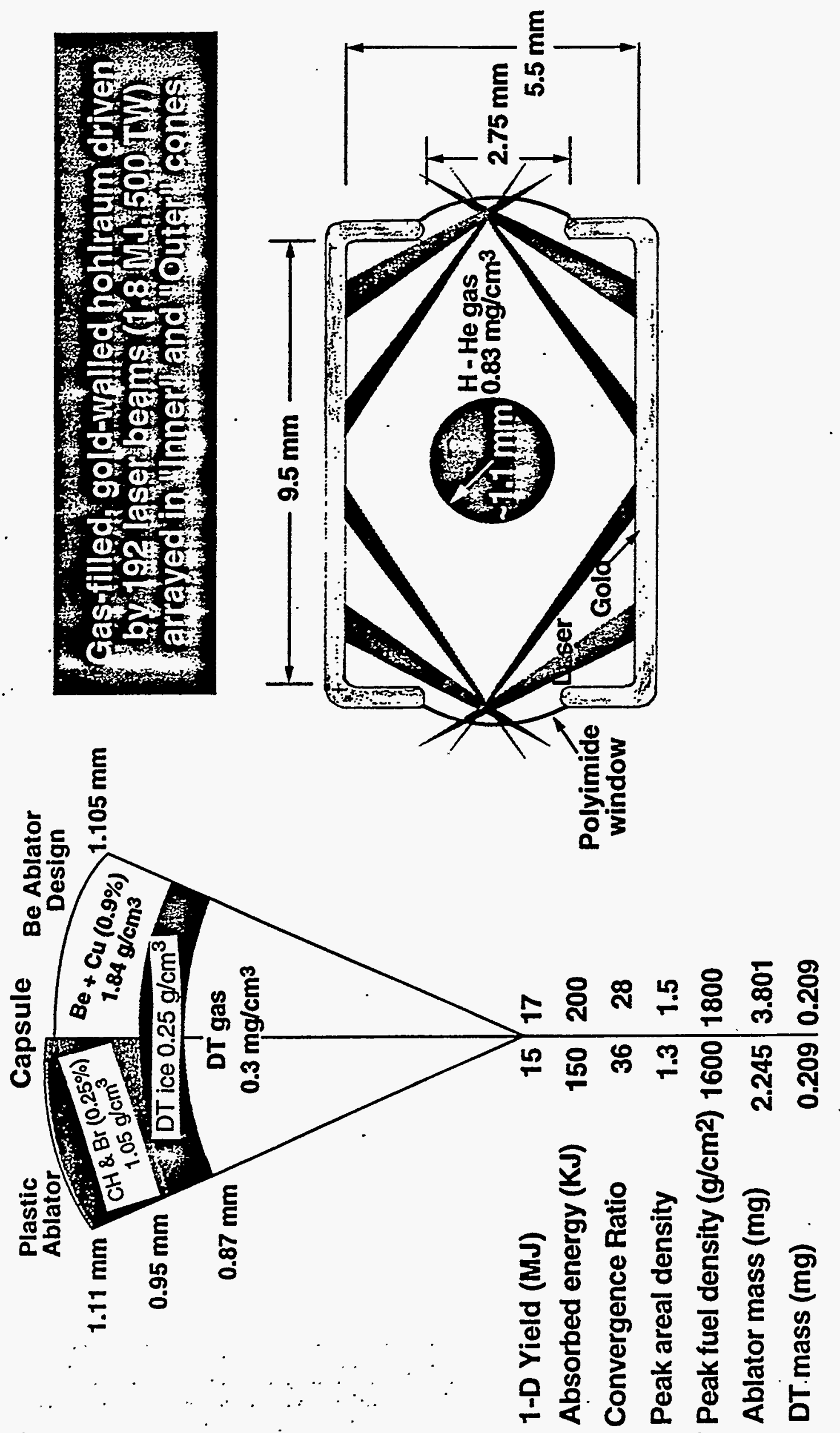


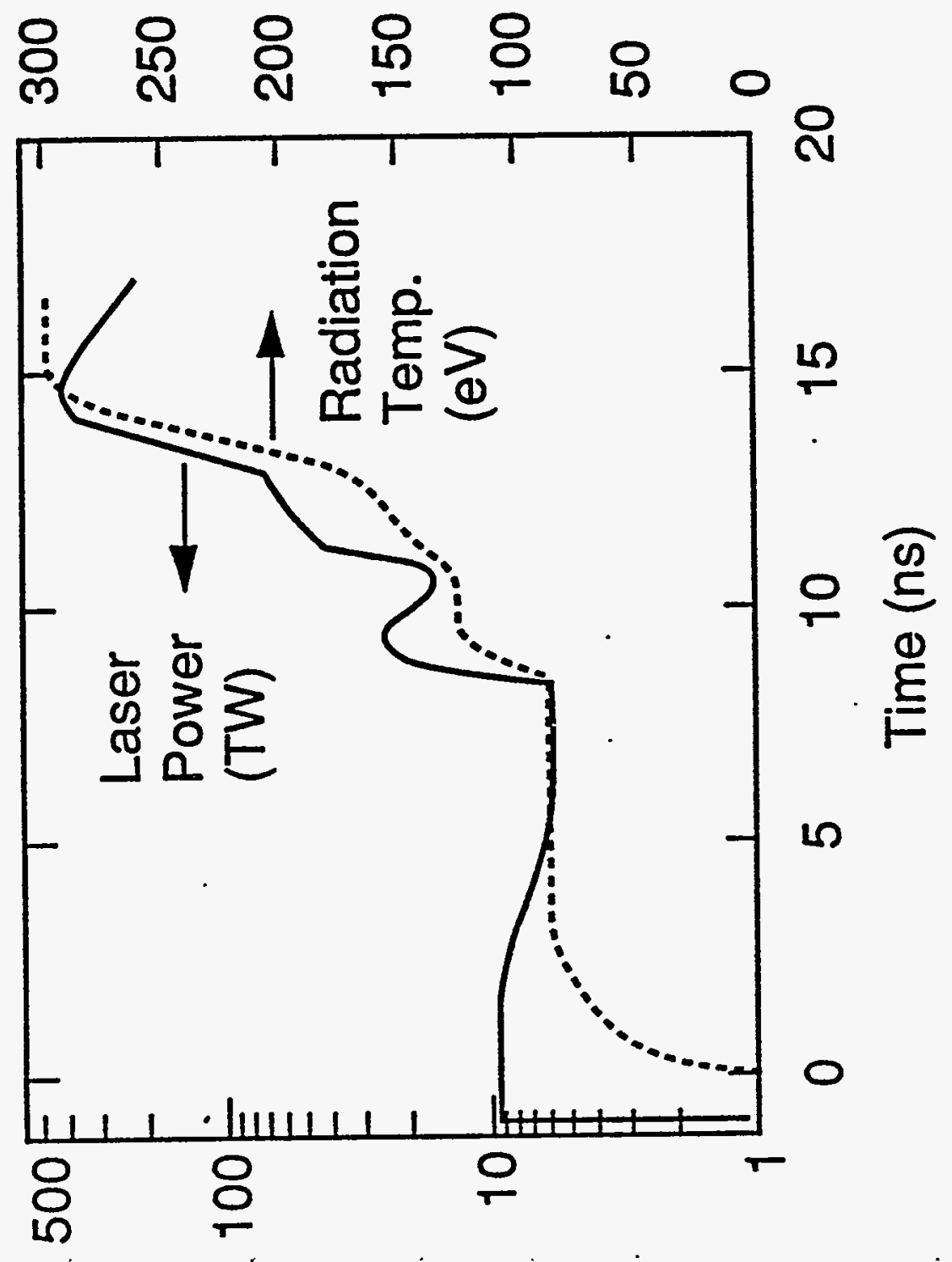



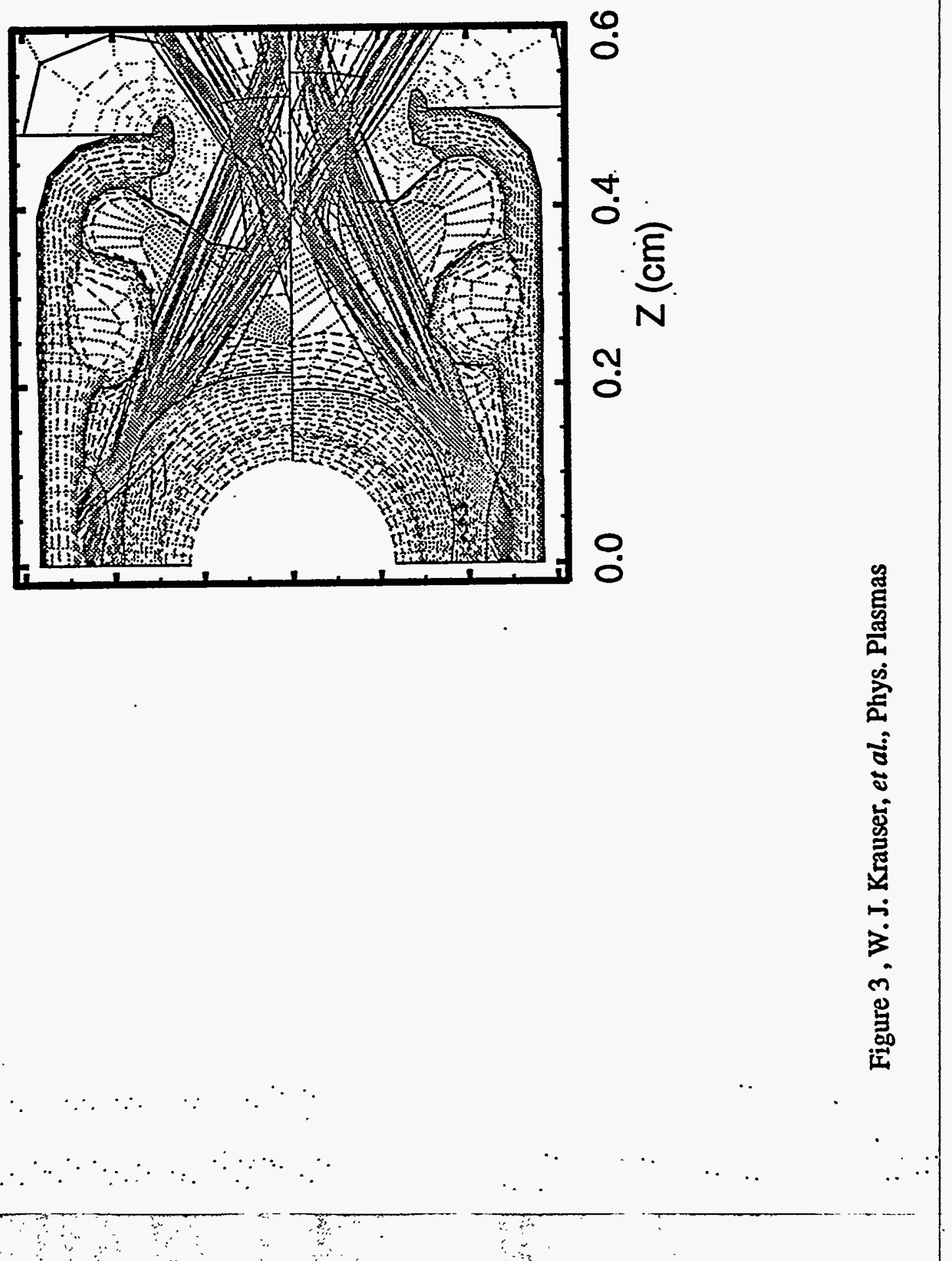


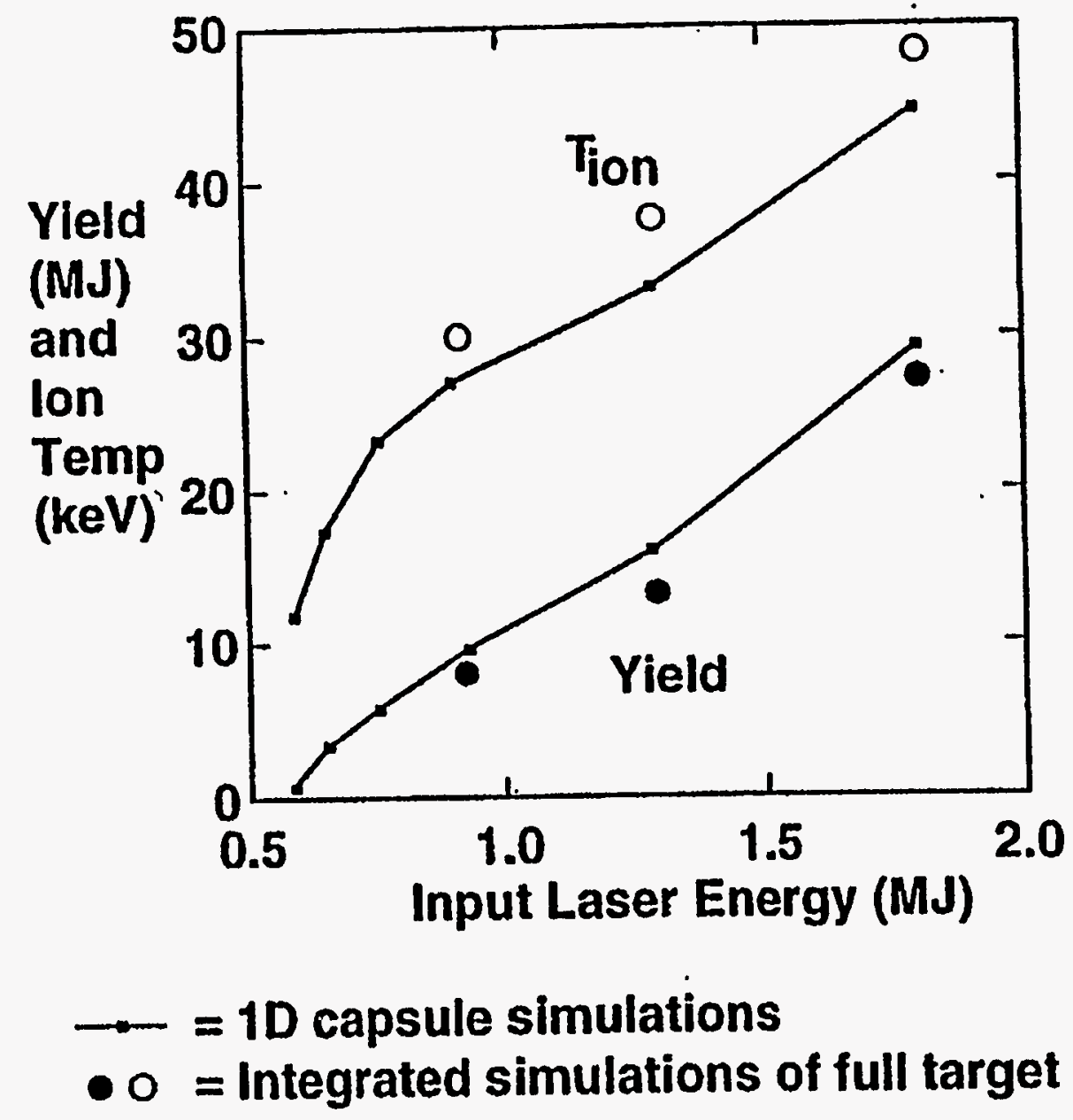




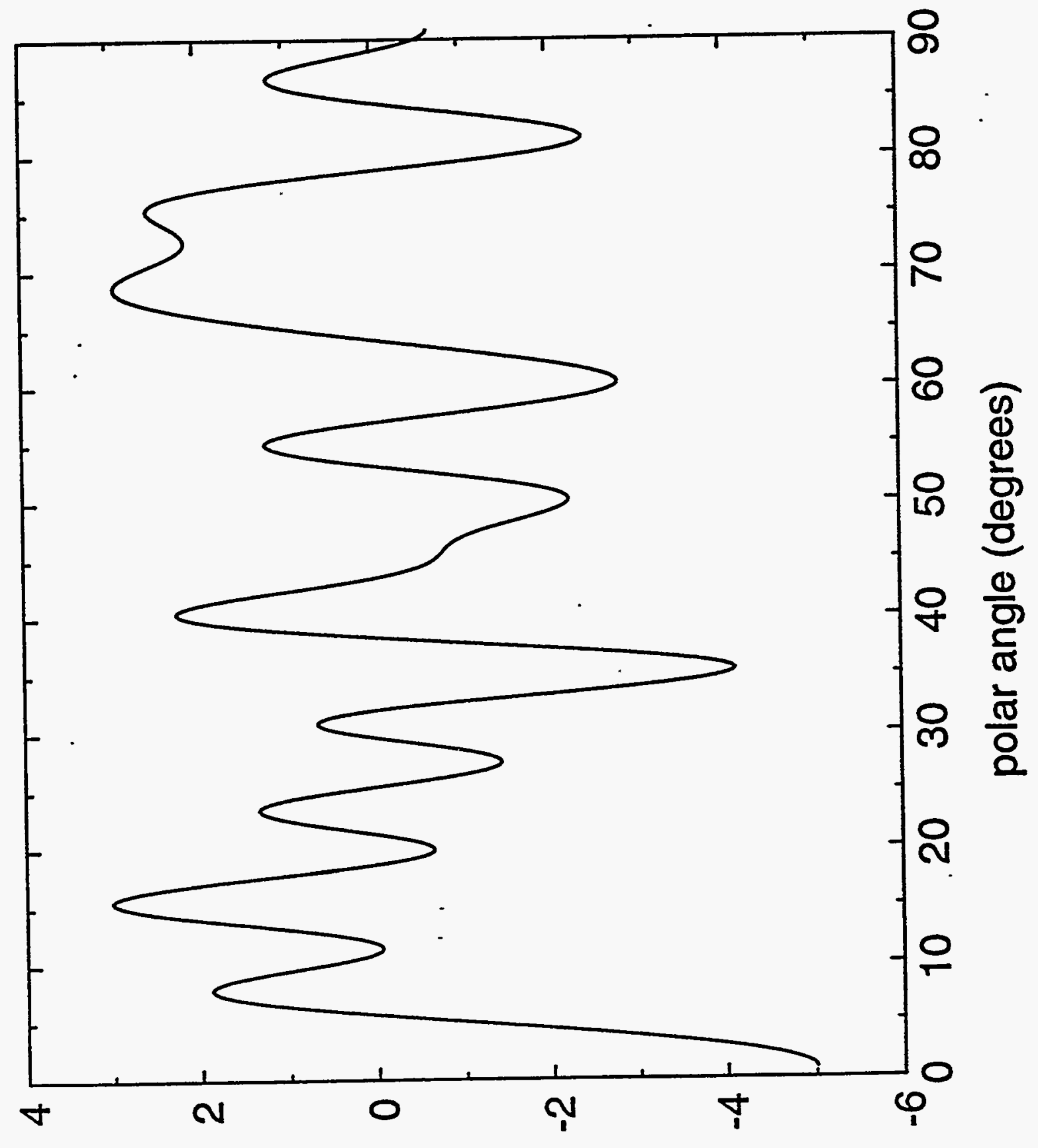

(uni) juəuəoejds!p je!ped 


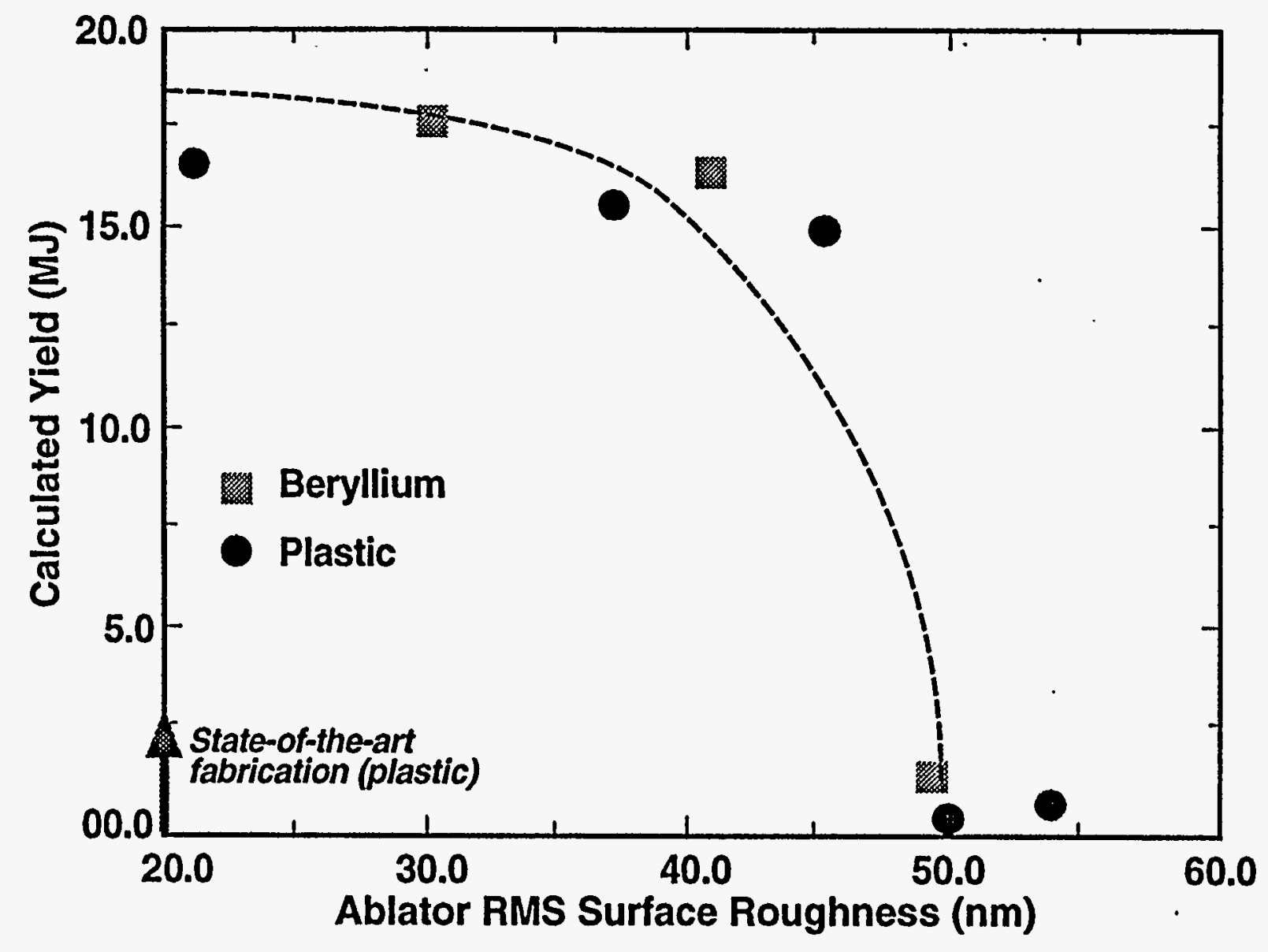

Figure 6, W. J. Krauser, et al., Phys. Plasmas 


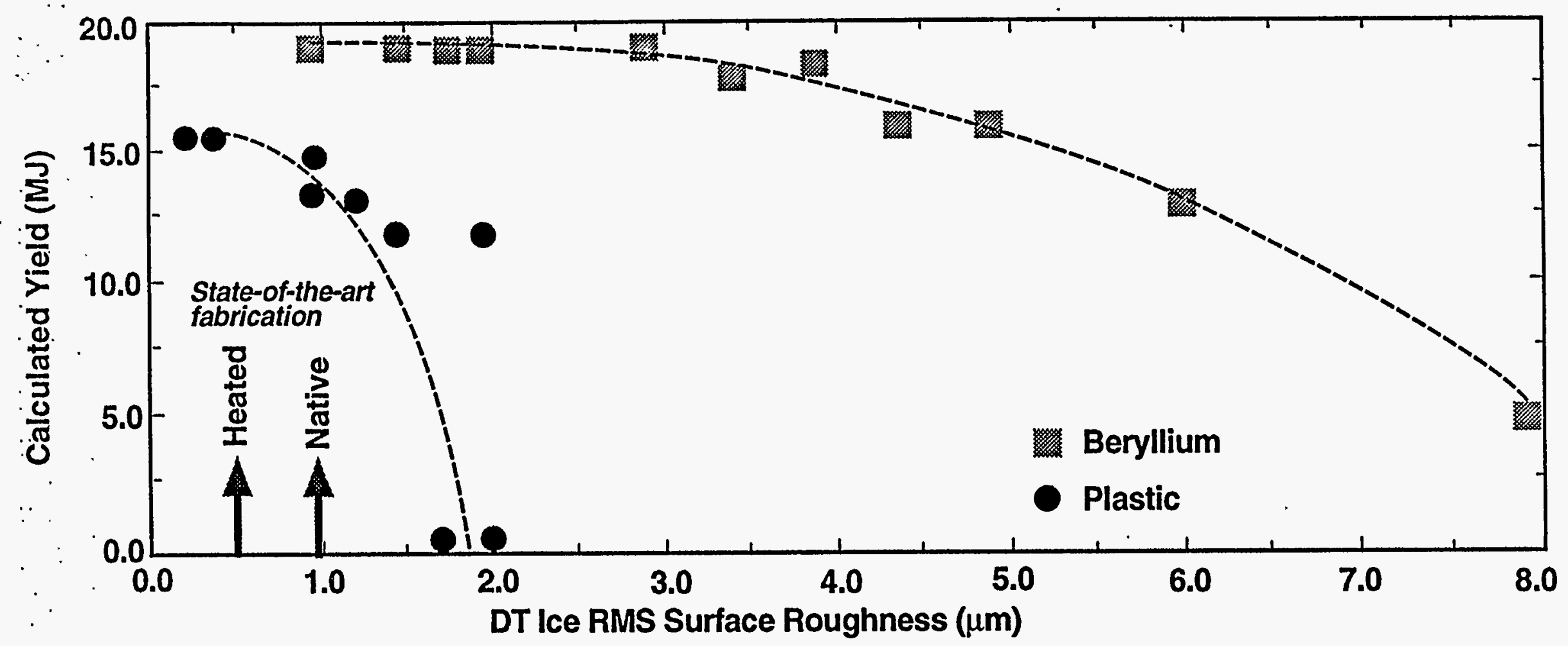

Figure 7 , W. J. Krauser, et al., Phys. Plasmas 


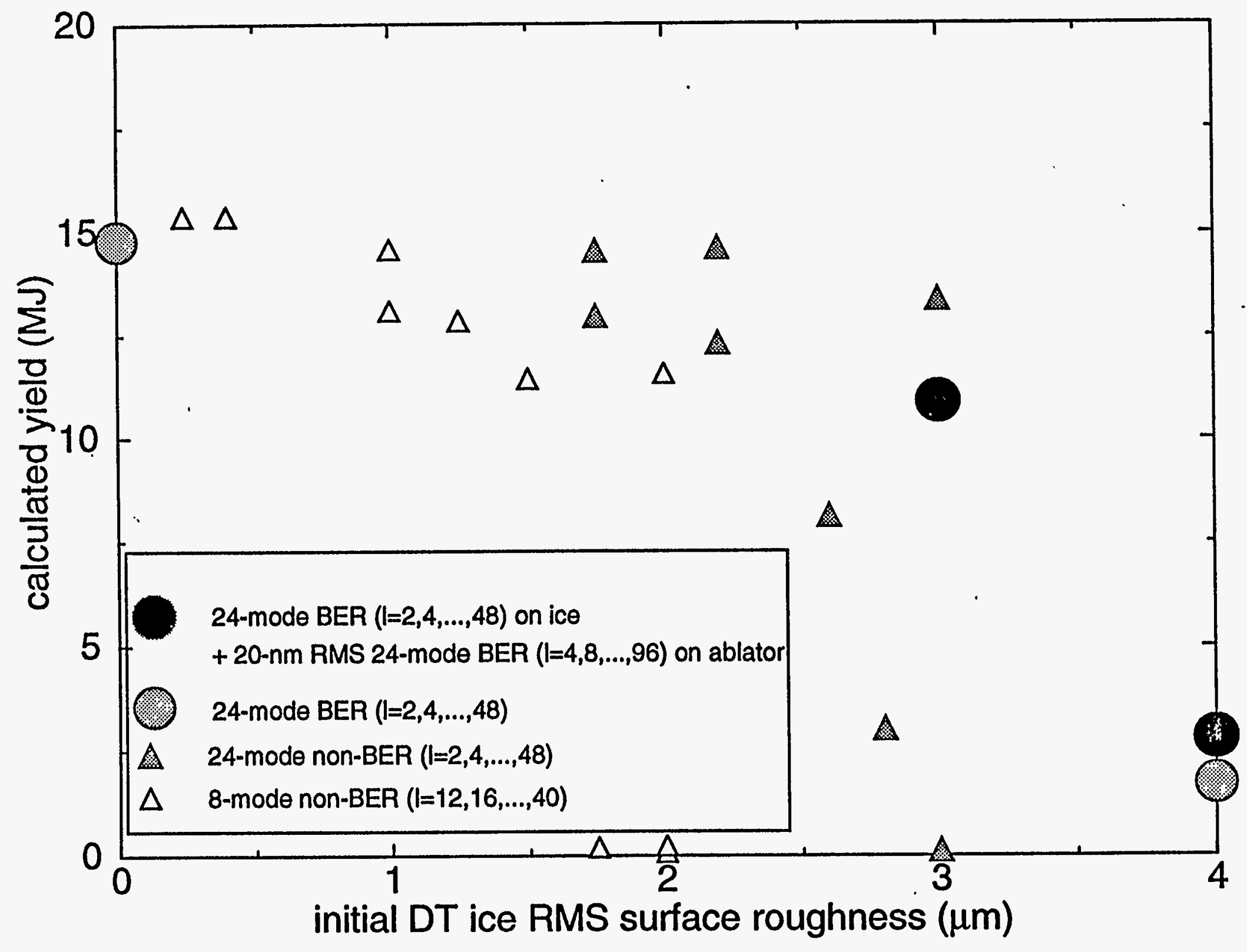

Figure 8 , W. J. Krauser, et al., Phys. Plasmas 


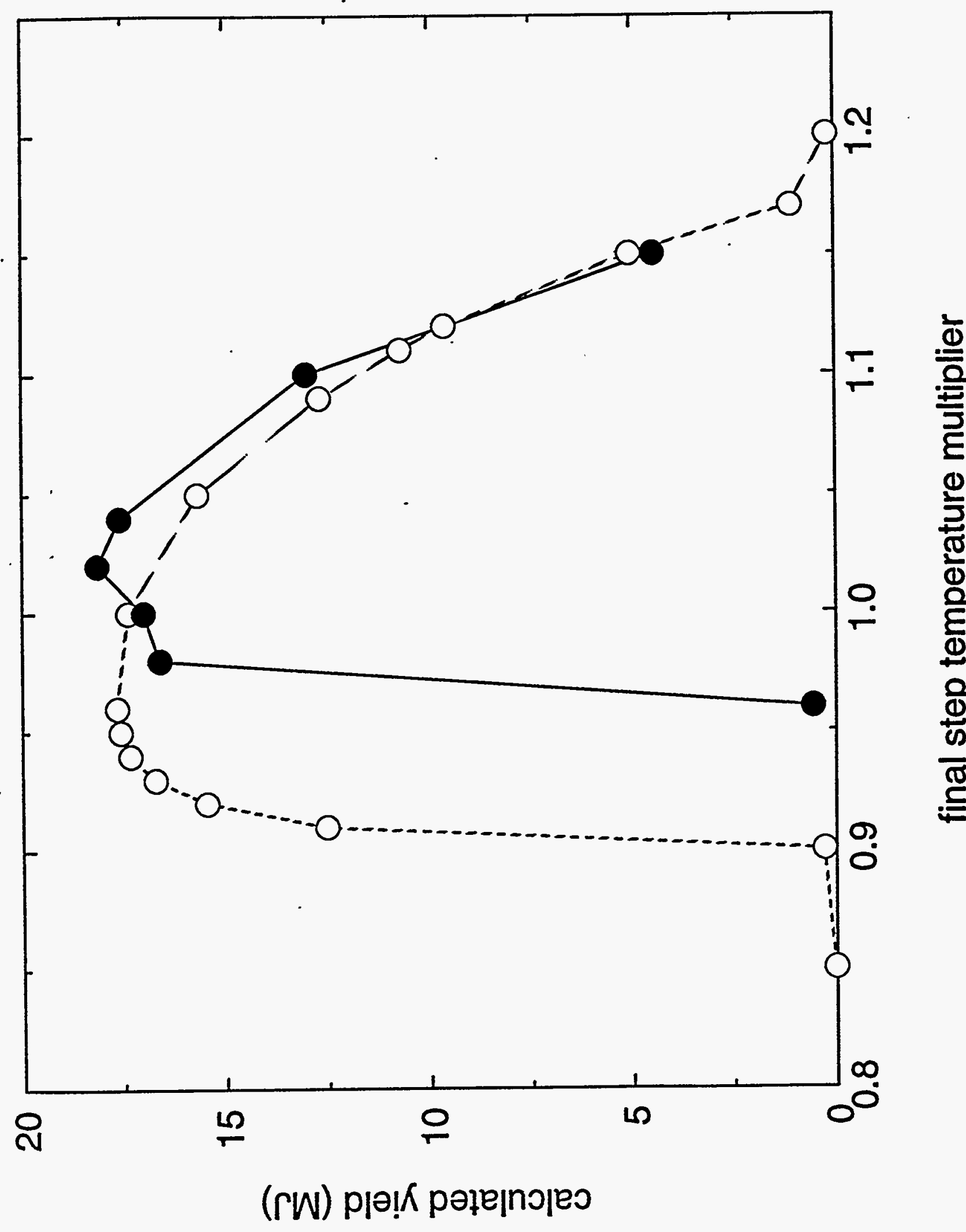

䓪 